\title{
Obsessive and Compulsive Characteristics of Alcohol Abuse and Dependence: Quantification by a Newly Developed Questionnaire
}

\author{
Jack G. Modell, Frederick B. Glaser, James M. Mountz, Stephen Schmaltz, and Louis Cyr
}

\begin{abstract}
The purpose of the this study was to develop an instrument for measuring the obsessive and compulsive characteristics of drinkingrelated thought and behavior in subjects who abuse or are dependent on alcohol, and to quantify the extent to which drinking-related thought and behavior in these subjects resemble the obsessions and compulsions seen in obsessive-compulsive disorder (OCD). To achieve these goals, the Yale-Brown Obsessive Compulsive Scale (Y-BOCS) was modified to reflect obsessionality and compulsivity specifically related to heavy drinking rather than to obsessions and compulsions generally. The modified Y-BOCS (Y-BOCS-hd) was administered to 62 subjects satisfying DSM-III-R criteria for alcohol abuse or alcohol dependence and 62 matched normal controls. The data showed that the Y-BOCS-hd is a sensitive and specific instrument for measuring the obsessive and compulsive characteristics of drinking-related thought and behavior in alcohol-abusing and alcohol-dependent populations, and that there are specific and quantifiable similarities between these characteristics and the obsessions and compulsions of OCD. The data also indicated that the Y-BOCShd may be a useful screening instrument for the presence of alcohol abuse and dependence.
\end{abstract}

Key Words: Obsessions, Compulsions, Alcoholism, ObsessiveCompulsive Disorder.

$\mathbf{S}^{1}$ IMILARITIES BETWEEN drinking-related thought and behavior observed in individuals who abuse alcohol and, respectively, the obsessions and compulsions observed in obsessive-compulsive disorder (OCD) have previously been noted. ${ }^{1-3}$ In OCD, the affected individual must struggle with the control or suppression of recurrent and persistent thoughts that often impel behaviors recognized by the individual as maladaptive or potentially destructive. This struggle resembles that faced by many heavy drinkers who experience strong urges or desires to drink - a struggle that, when lost, often leads to potentially destructive drinking behavior that may have "driven" or compulsive qualities. ${ }^{2,4-6}$ Past studies and discussions concerning the relationship between the thoughts and behaviors associated with heavy drinking, and those associated

From the Department of Psychiatry (J.G.M.), Department of Radiology (J.M.M.), and Department of Biostatistics and Biomathematics (L.C.), University of Alabama, Birmingham, Alabama; the Department of Psychiatry and Substance Abuse Center (F.B.G.), and Department of Internal Medicine, Clinical Research Center (S.S.), University of Michigan, Ann Arbor, Michigan.

Received for publication July 26, 1991; accepted November 20,1991

Reprint requests: Jack G. Modell, M.D., Department of Psychiatry, University of Alabama, Smolian Psychiatric Clinic, Room 403, UAB Station, Birmingham, AL 35294-0018.

Copyright $(1992$ by The Research Society on Alcoholism. with OCD, however, have been largely anecdotal or descriptive. Additionally, a review of the literature revealed no instruments for measuring this relationship quantitatively. Accordingly, the goals of the present study were to develop an instrument for measuring the obsessive and compulsive characteristics of drinking-related thought and behavior in individuals who abuse alcohol, and to quantify the extent to which this thought and behavior resembles the obsessions and compulsions seen in OCD.

We reasoned that if urges or desires to drink and heavy drinking in individuals who abuse or are dependent upon alcohol (henceforth, "alcoholic(s)") truly have the characteristics of obsessions and compulsions (respectively), then existing instruments for obsessionality and compulsivity specific for OCD should be sensitive to drinkingrelated thought and behavior in alcoholic individuals; additionally, these instruments should not be sensitive to drinking-related thought and behavior in the non-alcoholic individual. Furthermore, drinking-related responses by alcoholic individuals on this instrument should be of similar magnitude to those regarding obsessions and compulsions in the OCD population; to the extent that differences in responses between these populations emerge, they should help to clarify the relationship between alcoholrelated and OCD-related thought and behavior. Such an instrument might be useful in discriminating or screening alcoholic individuals from nonalcoholic individuals should it reveal large differences in measures of drinkingrelated obsessionality and compulsivity between alcoholic subjects and nonalcoholic controls.

\section{METHODS}

The Yale-Brown Obsessive Compulsive Scale (Y-BOCS), developed by Goodman and colleagues as a rating instrument for $\mathrm{OCD},{ }^{7,8}$ was chosen for modification to reflect obsessionality and compulsivity specifically related to heavy drinking rather than to obsessions and compulsions generally. The design of the Y-BOCS makes it especially well-suited for such modification with only minor changes since it "provides a specific measure of the severity of symptoms for obsessive-compulsive disorder that is not influenced by the type of obsessions or compulsions present." ${ }^{\prime}$ These changes were largely limited to replacing the terms "obsessive thoughts" with the phrase "ideas, thoughts, impulses, or images related to drinking"; and "compulsive behaviors" with "drinking alcoholic beverages." Additionally, the qualifier " 1 to 2 days after your last drink" was added to the questions pertaining to "ideas, thoughts, impulses, or images related to drinking" in order to provide a specific time reference for these questions. Only one question on the Y-BOCS, 
that which pertains to "time spent performing compulsions," did not readily lend itself to simple modification and was therefore rewritten and rescaled to reflect salience of drinking behavior as a function of daily and weekly drinking frequency. The numeric rating scales on all other questions were unchanged. The modified Y-BOCS, termed the YaleBrown Obsessive Compulsive Scale for heavy drinking (Y-BOCS-hd), is shown in the Appendix.

The experimental population was composed of 62 subjects ( 47 men, 15 women; mean age 39 , range 22 to 67) who satisfied DSM-III-R criteria for alcohol abuse or alcohol dependence and no other axis I disorder. Experimental subjects were in treatment for alcohol abuse at the University of Michigan or University of Alabama Psychiatric Outpatient Departments, or the Ann Arbor Veterans Administration Hospital (inpatient). The last drink of alcohol was 2 days to 4 weeks prior to study induction for all alcoholic subjects. The control population consisted of 62 subjects ( 47 men, 15 women; mean age 39 , range 24 to 69 ) who did not fulfill criteria for alcohol-dependence, abuse, or any other DSM-IIl$\mathrm{R}$ axis I disorder. The groups were age-, sex and education-matched. The Y-BOCS-hd was administered to all subjects by one of the investigators (JGM).

The responses of the alcoholic group for each question of the $Y$ BOCS-hd, as well as for the sum of questions 1 through 5 (obsessionality subscale), 6 through 10 (compulsivity subscale), and 1 through 10 were compared with those of the control group using the Mann-WhitneyWilcoxon rank sum test. Responses for each question of the Y-BOCShd in the alcoholic group were likewise compared with the published distribution of responses on the Y-BOCS in OCD ${ }^{8}$ Finally, the resulting frequency distributions of the Y-BOCS-hd subscale and full scale totals were analyzed to assess the degree of overlap of response totals between the alcoholic and control groups. Numeric cut-points in these distributions were obtained by discriminant function analysis and count statistics to maximize the discriminant power of the Y-BOCS-hd questions. Nonparametric statistical procedures were used because of the ordinal nature of the Y-BOCS-hd data. To provide a conservative interpretation of results, we adopted a significance level of $\alpha<0.01$ for all comparisons.

\section{RESULTS}

The mean scores for each question on the Y-BOCS-hd, and for the Y-BOCS-hd questions 1 through 5, 6 through 10 , and 1 through 10 sums were all significantly greater in the alcoholic group than in the normal controls $(\mathbb{Z} \mid \geq 8.5$, $p \leq 0.0001$ for each; Table 1). In contrast, with the exception of question 6 , which is specific to time engaged in drinking or compulsive behaviors, there were no signif-

Table 1. Scores on an Existing Instrument for Obsessionality and Compulsivity Specific for OCD, Modified to Reflect Drinking-Related Thought and Behavior for Alcoholic and Nonalcoholic Individuals (Y-BOCS-hd)

\begin{tabular}{|c|c|c|}
\hline $\begin{array}{l}\text { Y-BOCS-hd } \\
\text { Question }\end{array}$ & $\begin{array}{c}\text { Alc } \\
\bar{x}(S D)\end{array}$ & $\begin{array}{l}\text { Control } \\
\bar{x}(S D)\end{array}$ \\
\hline $1^{* * *}$ & $2.2(1.1)$ & $0.06(0.23)$ \\
\hline $2^{* \star \star}$ & $1.4(0.96)$ & $0.02(0.14)$ \\
\hline $3^{\star \star \star *}$ & $1.9(1.1)$ & $0.02(0.14)$ \\
\hline $4^{* * \star}$ & $1.9(1.2)$ & $0.04(0.19)$ \\
\hline $5^{\star \star \star}$ & $2.1(1.1)$ & $0.05(0.20)$ \\
\hline $6^{* * *}$ & $3.3(0.86)$ & $0.63(0.70)$ \\
\hline $7^{\star \star *}$ & $2.0(1.2)$ & $0.05(0.20)$ \\
\hline $8^{\star * *}$ & $2.5(1.2)$ & $0.12(0.32)$ \\
\hline $9^{* \star *}$ & $2.5(1.2)$ & $0.11(0.42)$ \\
\hline $10^{* * \hbar}$ & $2.6(0.96)$ & $0.07(0.26)$ \\
\hline $1-5$ sum $^{* * *}$ & $9.6(4.3)$ & $0.16(0.55)$ \\
\hline 6-10 sum*** & $12.9(4.1)$ & $0.94(1.5)$ \\
\hline $1-10$ sum**** & $22.3(7.7)$ & $1.1 \quad(1.8)$ \\
\hline
\end{tabular}

The mean scores $(\bar{x})$ and standard deviations $(S D)$ for each question on the $Y$. BOCS-hd and for the $Y$-BOCS-hd question $1-5,6-10$, and 1-10 sums are shown for the alcoholic (AlC) and control groups. ${ }^{* * *} p \leq 0.0001$. icant differences between mean scores on the Y-BOCS-hd compulsivity subscale in the alcoholic group and those previously published for subjects with $O C D$ on the YBOCS (Table 2). ${ }^{8}$ Reported scores for question 6 were greater in the alcoholic population than those published for OCD $(Z \mid=2.8, p<0.01)$. The mean scores for all questions on the obsessionality subscale, however, were significantly lower in the alcoholic group than the OCD population $([Z] \geq 2.8, p<0.01)$ with the exception of question 4 regarding resistance against alcohol related thoughts $(|Z|=1.0, p=0.3)$.

Choosing numeric cut-points in the Y-BOCS-hd subscale and full scale frequency distributions by discriminant analysis yielded classification probabilities of $94 \%$ to $98 \%$ for subjects by group, as shown in Table 3 . Of note, the responses to question 6 alone (pertaining solely to reported quantity/frequency of alcohol use), yielded correct classification of $98 \%$ of the alcoholics and $89 \%$ of the nonalcoholic controls using a cut-point of 2.0 (maximized sensitivity); and $98 \%$ of the controls and $78 \%$ of the alcoholics using a cut-point of 2.5 (maximized specificity). Sample

Table 2. Similarities and Differences between Drinking-Related Responses by Alcoholic Individuals and Responses Regarding Obsessions and Compulsions in an OCD Population

\begin{tabular}{cll}
\hline $\begin{array}{c}\text { Y-BOCS-hd/ } \\
\text { Y-BOCS } \\
\text { Questions }\end{array}$ & \multicolumn{1}{c}{$\begin{array}{c}\text { Alc } \\
\overline{\mathrm{x}}(\mathrm{SD})\end{array}$} & \multicolumn{1}{c}{$\begin{array}{c}\text { OCD } \\
\overline{\mathrm{x}}(\mathrm{SD})\end{array}$} \\
\hline $1^{*}$ & $2.2(1.1)$ & $2.8(1.0)$ \\
$2^{*}$ & $1.4(0.96)$ & $2.2(0.86)$ \\
$3^{*}$ & $1.9(1.1)$ & $2.4(1.0)$ \\
4 & $1.9(1.2)$ & $2.1(1.1)$ \\
$5^{*}$ & $2.1(1.1)$ & $2.8(0.78)$ \\
$6^{*}$ & $3.3(0.86)$ & $2.8(0.96)$ \\
7 & $2.0(1.2)$ & $2.3(0.97)$ \\
8 & $2.5(1.2)$ & $2.6(0.91)$ \\
9 & $2.5(1.2)$ & $2.5(1.1)$ \\
10 & $2.6(0.96)$ & $2.9(0.91)$ \\
\hline
\end{tabular}

The mean scores $(\bar{x})$ and standard deviations (SD) for each question on the $Y$ BOCS-hd for the alcoholic subjects and those previously published for subjects with OCD on the Y-BOCS are shown. ${ }^{*} p \leq 0.01$.

Table 3. Sensitivities, Specificities, and Predictive Values for Classification of Individual Subjects as Either Normal or Alcoholic Based on Specified Cut-Points in the Y-BOCS-hd Subscale and Full Scale Totals (Alcoholic $\geq$ Cut-Point)

\begin{tabular}{|c|c|c|c|c|c|}
\hline Measures & $\begin{array}{l}\text { Cut- } \\
\text { Point }\end{array}$ & $\begin{array}{l}\% \text { Correctly } \\
\text { Classified } \\
\text { as Alcoholic } \\
\text { (Sensitivity) }\end{array}$ & $\begin{array}{l}\% \text { Correctly } \\
\text { Classified } \\
\text { as Normal } \\
\text { Control } \\
\text { (Specificity) }\end{array}$ & $\begin{array}{c}\text { Positive- } \\
\text { Predictive } \\
\text { Value for } \\
\text { Alcoholism } \\
(\%)\end{array}$ & $\begin{array}{c}\text { Negative } \\
\text { Predictive } \\
\text { Value } \\
\text { (Nonalcoholic) } \\
(\%)\end{array}$ \\
\hline $\begin{array}{l}\text { Y-BOCS-hd } \\
1-5 \text { sum }\end{array}$ & 2.0 & 97 & 95 & 78 & 99 \\
\hline $\begin{array}{l}\text { Y-BOCS-hd } \\
6-10 \text { sum }\end{array}$ & 5.0 & 98 & 94 & 74 & 99 \\
\hline $\begin{array}{l}\text { Y-BOCS-hd } \\
1-10 \text { sum }\end{array}$ & 7.0 & 96 & 98 & 89 & 99 \\
\hline $\begin{array}{l}\text { Y-BOCS-hd } \\
6 \text { alone }\end{array}$ & 2.0 & 98 & 89 & 61 & 99 \\
\hline $\begin{array}{l}\text { Y-BOCS-hd } \\
6 \text { alone }\end{array}$ & 2.5 & 78 & 98 & 87 & 96 \\
\hline
\end{tabular}

Sensitivities and specificities are derived directly from the experimental data. Predictive values are calculated based on a population-prevalence of $15 \%$ for combined alcohol dependence and abuse (alcoholism). Information from analysis of the response distributions of question 6 alone is also shown; note that the numeric cut-points in the Y-BOCS-hd can be adjusted as needed to optimize specificity or sensitivity. 
size limitations did not permit valid assessment of age or sex differences in the outcome measures.

\section{DISCUSSION}

The nonzero, near midscale scores on all questions of the Y-BOCS-hd in the alcoholic population indicate that this instrument is sensitive to drinking-related thought and behavior in this population. In contrast, the near-zero scores on all Y-BOCS-hd questions in the control population (except question 6; see below) indicates that the $\mathrm{Y}$ BOCS-hd is also specific for drinking-related thought and behavior in the alcoholic population; there are essentially no obsessive or compulsive features to alcohol-related thought or behavior in the nonalcoholic. It is, of course, possible that answers similar to those obtained on the YBOCS-hd in the alcoholic population might also be obtained from individuals suffering from other addictive disorders on appropriately rephrased versions of the YBOCS. The Y-BOCS-hd question 6 mean score of 0.63 in the nonalcoholic controls simply indicates a reported average quantity/frequency of alcohol drinking in this group of less than one drink daily and one or less drinking day weekly.

Mean scores on the Y-BOCS-hd compulsive subscale in the alcoholic population were comparable with mean scores on this subscale in the OCD population, suggesting that drinking-related behavior in the alcoholic individual is similar in nature and degree to the compulsions of OCD. These similarities specifically include: impaired social or occupational function resulting from performance of the behavior (drinking or compulsions; Y-BOCS/YBOCS-hd question 7); moderate to severe subjective distress associated with the behavior (question 8); inconsistent or partial effort made to resist the behavior (question 9); and impaired control over the behavior (question 10). The mean score of 3.3 for Y-BOCS-hd question 6 in the alcoholic population indicates that drinking in alcoholic individuals, like performance of compulsions in individuals with $O C D$, is a salient or heavily time-consuming activity. The required changes from the Y-BOCS to the $\mathrm{Y}$-BOCS-hd in the wording and scaling of this question, however, preclude direct comparisons of time spent drinking in the alcoholic population with time spent performing compulsions in the OCD population.

Mean scores for all but one of the Y-BOCS/Y-BOCShd obsessional subscale questions were, however, significantly less in the alcoholic than in the OCD comparison group. These differences are: frequency of thought intrusion (question 1), degree of impairment in social or occupational function resulting from thought intrusion (question 2), amount of distress associated with thought intrusion (question 3), and degree of control over these thoughts (question 5). The means for Y-BOCS/Y-BOCShd question 4 , the amount of effort made to resist intrusive thoughts, did not significantly differ between the alcoholic and OCD groups. Although the means on this subscale for the alcoholic group were all in the mild-to-moderate range of severity, the lower Y-BOCS/Y-BOCS-hd obsessional subscale means in the alcoholic group than in the OCD group indicate that intrusive thoughts about drinking are less pervasive and disturbing than are the obsessions of OCD. This relative difference has been noted anecdotally in the past and, in itself, has been used as an argument against a specific relationship between OCD and alcoholism.

Another important difference between the obsessions of OCD and drinking-related thought in alcoholism that has also been used to argue against a true pathogenetic relationship between these disorders is seen in the following example noted by Caetano. ${ }^{1}$ Unlike the repetitive urge of an individual with OCD to injure her child, he notes, the urge to drink in the alcoholic is less likely to be viewed by the individual as senseless and more likely to be seen as a need that begs to be satisfied. We submit, however, that this apparent difference may be rooted in cultural norms regarding thought and behavior. While an individual might well have ambivalent or hostile feelings toward a loved one, society dictates that these feelings are not acceptable and should be suppressed or denied; furthermore, acting on the resultant impulses might be a criminal offense. In contrast, we are rarely taught that the desire to drink is inherently bad, and our society often promotes drinking as desirable or glamorous, despite its well-known destructive potential. This distinction between obsessional thought in OCD and repetitive urges to drink in alcoholism may, therefore, be more a reflection of the cognitive ("cortical") processing of intrusive thoughts or impulses than indicative of a fundamental difference in the pathogenesis of these disorders. A similar subcortical basis for alcoholism and OCD has been previously proposed. ${ }^{3,9}$ The design and results of the current study do not, however, permit the conclusion that alcoholism and OCD are simply different manifestations of the same disorder; drinkingrelated thought and behavior in alcoholism and the obsessions and compulsions of OCD may differ substantially in ways not measured by the Y-BOCS-hd.

Support for the potential usefulness of the Y-BOCS-hd in discriminating or screening alcoholic individuals from nonalcoholic individuals is very strong; there was almost no overlap of ratings on the Y-BOCS-hd questions between the alcoholic and normal control groups. These nonoverlapping distributions allow for high sensitivies, specificities, and predictive values in distinguishing alcoholic from nonalcoholic individuals based on responses to the Y-BOCS-hd questions alone (Table 3). The discriminant power found for the Y-BOCS-hd exceeds that published for the CAGE and MAST questionnaires, both widely used as screening tests for alcoholism. ${ }^{10-12}$ It should be recalled, however, that the alcoholic subjects who completed this questionnaire had recently entered a psychiatric treatment program and in many cases had already ac- 
knowledged that a drinking problem might exist. Whether the Y-BOCS-hd will have similar prospective discriminative power in the general population and in individuals who drink heavily but do not meet DSM-III-R criteria for alcohol dependence or abuse remains to be tested.

One potential advantage of the Y-BOCS-hd over the CAGE and MAST questionnaires is that, unlike the latter questionnaires, scoring on the Y-BOCS-hd does not rely upon restricted or environment-dependent consequences of drinking such as arrests, withdrawal symptoms, or others being annoyed by the drinking. The Y-BOCS-hd also addresses the salience of drinking-related thought and behavior (in particular, questions 1, 2, 6, and 7) over other, more consequential effects of heavy drinking; salience of drink-seeking behavior is, in fact, considered by Edwards and Gross ${ }^{2}$ and Edwards ${ }^{13}$ to be an essential element of the alcohol-dependence syndrome. The predictive power of question 6 alone is noteworthy in this regard. While both alcoholic and nonalcoholic individuals might under-report the quantity and frequency of their drinking, these data show that self-reports of how much and how frequently one drinks alcohol may, nonetheless, discriminate with relative accuracy between alcoholic and nonal- coholic individuals by using empirically derived cut-points in this self-report scale.

The results and conclusions of this study should be viewed as somewhat preliminary in nature since the somewhat limited sample size precludes unequivocal validation and reliability-testing of the Y-BOCS-hd. It does, however, appear that the Y-BOCS-hd may allow quantification of the obsessive and compulsive features of heavy drinking in the alcoholic individual. This instrument could, therefore, be useful in enhancing our understanding of the role of these cognitive and behavioral features in heavy drinking and its treatment, especially if Y-BOCS-hd scores can be shown to discriminate among heavy drinkers on the basis of important pathogenetic or prognostic factors. Age and gender differences on the Y-BOCS-hd that might emerge from a larger sample size will also be of particular interest. Finally, the minimal overlap of the Y-BOCS-hd scores from the alcoholic and nonalcoholic control groups indicate that the Y-BOCS-hd may be a useful screening instrument for alcohol abuse and dependence.

\section{ACKNOWLEDGMENT}

The authors gratefully acknowledge the editorial assistance of Cynthia S. Pomerleau, Ph.D.

Yale-Brown Obsessive Compulsive Scale Modified to Reflect Obsessions and Compulsions Related to Heavy Drinking (Y-BOCS-hd)

Obsessive subscale, questions 1-5

\section{TIME OCCUPIED BY ALCOHOL-RELATED} THOUGHTS

Q: How much of your time when you've gone without a drink for 1 to 2 days is occupied by ideas, thoughts, impulses, or images related to drinking? How frequently do these thoughts occur?

0 - None

1 - Mild (less than $1 \mathrm{hr}$ per day), or occasional intrusion (occur no more than 8 times a day)

2 - Moderate ( 1 to $3 \mathrm{hr}$ per day), or frequent intrusion (occur more than 8 times a day, but most hours of the day are free of these thoughts)

3 - Severe (greater than 3 and up to 8 hr per day), or very frequent intrusion (occur more than 8 times a day and occur during most hours of the day)

4 - Extreme (greater than $8 \mathrm{hr}$ per day), or near constant intrusion (too numerous to count and an hour rarely passes without several such thoughts occurring)

2. INTERFERENCE DUE TO ALCOHOL-RELATED THOUGHTS

Q: How much do the ideas, thoughts, impulses, or images related to drinking interfere with your social or work (or role) functioning 1 to 2 days after your last drink? Is there anything you don't do because of them? (If currently not working, determine how much performance would be affected if subject were employed.)

0 - None

1 - Mild, slight interference with social or occupational activities, but overall performance not impaired

2 - Moderate, definite interference with social or occupational performance, but still manageable

3 - Severe, causes substantial impairment in social or occupational performance

4 - Extreme, incapacitating

3. DISTRESS ASSOCIATED WITH ALCOHOL-RELATED THOUGHTS

Q: How much distress do these ideas, thoughts, impulses, or images related to drinking cause you when you've gone without a drink for 1 to 2 days? (In most cases, distress is equated with anxiety; however, subjects may report that these thoughts are "disturbing" but deny "anxiety." Only rate anxiety that seems triggered by these thoughts, not generalized anxiety or anxiety associated with other symptoms.)

0 - None

1 - Mild, infrequent, and not too disturbing 
2 - Moderate, frequent, and disturbing, but still manageable

3 - Severe, very frequent, and very disturbing

4 - Extreme, near constant, and disabling distress

4. RESISTANCE AGAINST ALCOHOL-RELATED THOUGHTS

Q: How much of an effort do you make to resist these thoughts when you've gone without a drink for 1 to 2 days? How often do you try to disregard or turn your attention away from these thoughts as they enter your mind when you've gone without a drink for 1 to 2 days? (Only rate effort made to resist, not success or failure in actually controlling these thoughts. How much the subject resists these thoughts may or may not correlate with his/her ability to control them. Note that this question does not directly measure the severity of the intrusive thoughts; rather it rates a manifestation of health, i.e., the effort the subject makes to counteract these thoughts. Thus, the more the subject tries to resist, the less impaired is this aspect of his/her functioning. If these thoughts are minimal, the subject may not feel the need to resist them. In such cases, a rating of " 0 " should be given.)

0 - Makes an effort to always resist, or symptoms so minimal, doesn't need to actively resist

1 - Tries to resist most of the time

2 - Makes some effort to resist

3 - Yields to all such thoughts without attempting to control them, but does so with some reluctance

4 - Completely and willingly yields to all such thoughts

5. DEGREE OF CONTROL OVER ALCOHOL-RELATED THOUGHTS

Q: How much control do you have over these thoughts when you've gone without a drink for 1 to 2 days? How successful are you in stopping or diverting such thinking? (In contrast to the preceding question on resistance, the ability of the subject to control his/her thoughts is more closely related to the severity of the intrusive thoughts.)

0 - Complete control

1 - Much control, usually able to stop or divert such thoughts with some effort and concentration

2 - Moderate control, sometimes able to stop or divert such thoughts

3 - Little control, rarely successful in stopping such thoughts, can only divert attention with difficulty

4 - No control, experienced as completely involuntary, rarely able to divert thinking even momentarily

Compulsive subscale, questions $6-10$
6. TIME SPENT PERFORMING DRINKING BEHAVIORS

Q: How many drinks do you drink each day? How many days each week to do you drink? (Score as the maximum category for which both quantity and frequency apply. For example, four drinks daily 3 days weekly $=2$; four drinks daily 6 days weekly $=3$.)

0 - None (abstains)

1 - Mild, drinks less than one drink per day, and drinks no more than 1 day weekly

2 - Moderate, drinks one or more drinks per day, and drinks 2 or more days weekly

3 - Severe, drinks three or more drinks per day, and drinks 4 or more days weekly

4 - Extreme, drinks eight or more drinks per day, and drinks 6 or more days weekly

7. INTERFERENCE DUE TO ALCOHOLIC DRINKING

Q: How much does your drinking of alcoholic beverages interfere with your social or work (or role) functioning? Is there anything that you don't or can't do because of your drinking? (If currently not working, determine how much performance would be affected if subject were employed.)

0 - None

1 - Mild, slight interference with social or occupational activities, but overall performance not impaired

2 - Moderate, definite interference with social or occupational performance, but still manageable

3 - Severe, causes substantial impairment in social or occupational performance

4 - Extreme, incapacitating

8. DISTRESS ASSOCIATED WITH CONSUMPTION OF ALCOHOLIC BEVERAGES

Q: How would you feel if prevented from drinking alcohol when you desired a drink? (Pause) How anxious or upset would you become? (Rate degree of distress subject would experience if drinking of alcohol were suddenly interrupted without reassurance offered. In most, but not all cases, drinking behavior reduces anxiety.)

0 - None

1 - Mild, only slightly anxious or irritated if drinking prevented

2 - Moderate, reports that anxiety or irritation would mount but remain manageable if drinking prevented

3 - Severe, prominent and very disturbing increase in anxiety or irritation if drinking interrupted

4 - Extreme, incapacitating anxiety or irritation from any intervention aimed at modifying drinking 


\section{RESISTANCE AGAINST CONSUMPTION OF AL- COHOLIC BEVERAGES}

Q: How much of an effort do you make to resist drinking alcoholic beverages? (Only rate effort made to resist, not success or failure in actually controlling the drinking. How much the subject resists drinking may or may not correlate with his ability to control it. Note that this question does not directly measure the severity of drinking; rather it rates a manifestation of health, i.e., the effort the subject makes to counteract drinking. Thus, the more the subject tries to resist, the less impaired is this aspect of his functioning. If the drinking is minimal, the subject may not feel the need to resist it. In such cases, a rating of " 0 " should be given.)

0 - Makes an effort to always resist, or drinking so minimal doesn't need to actively resist

1 - Tries to resist most of the time

2 - Makes some effort to resist

3 - Yields to almost all drinking without attempting to control it, but does so with some reluctance

4 - Completely and willingly yields to all drinking

10. DEGREE OF CONTROL OVER CONSUMPTION OF ALCOHOLIC BEVERAGES

Q: How strong is the drive to drink alcoholic beverages? (Pause) How much control do you have over the drinking? (In contrast to the preceding question on resistance, the ability of the subject to control drinking is more closely related to the severity of drinking.)

0 - Complete control

1 - Much control, experiences pressure to drink, but usually able to exercise voluntary control over it

2 - Moderate control, strong pressure to drink, can control it only with difficulty

3 - Little control, very strong drive to drink, must be carried to completion, can only delay with difficulty

4 - No control, drive to drink experienced as completely involuntary and overpowering, rarely able to delay drinking even momentarily

\section{REFERENCES}

1. Caetano R: Alcohol dependence and the need to drink: A compulsion? Psychol Med 15:463-469, 1985

2. Edwards G, Gross MM: Alcohol dependence: Provisional description of a clinical syndrome. $\mathrm{Br}$ Med J 1:1058-1061, 1976

3. Modell JG, Mountz JM, Beresford TP: Basal ganglia/limbic striatal and thalamocortical involvement in craving and loss of control in alcoholism. J Neuropsychiatry Clin Neurosci 2:123-144, 1990

4. Jellinek EM: The Disease Concept of Alcoholism. Highland Park, NJ, Hill-house Press, 1960

5. Keller M: On the loss-of-control phenomenon in alcoholism. $\mathrm{Br}$ J Addict 67:153-166, 1972

6. Ludwig AM, Wikler A: "Craving" and relapse to drink. Q J Stud Alcohol 35:108-130, 1974

7. Goodman WK, Price LH, Rasmussen SA, Mazure C, Delgado P, Heninger GR, Charney DS: The Yale-Brown Obsessive Compulsive Scale: II. Validity. Arch Gen Psychiatry 46:1012-1016, 1989

8. Goodman WK, Price LH, Rasmussen SA, Mazure C, Fleischmann RL, Hill CL, Heninger GR, Charney DS: The Yale-Brown Obsessive Compulsive Scale: I. Development, use, and reliability. Arch Gen Psychiatry 46:1006-1011, 1989

9. Modell JG, Mountz JM, Curtis GC, Greden JF: Neurophysiologic dysfunction in basal ganglia/limbic striatal and thalamocortical circuits as a pathogenetic mechanism of obsessive-compulsive disorder. $\mathbf{J}$ Neuropsychiatry Clin Neurosci 1:27-36, 1989

10. Bush B, Shaw S, Cleary P, Delbanco TL, Aronson MD: Screening for alcohol abuse using the CAGE questionnaire. Am J Med 82:231235,1987

11. Mayfield D, McLeod G, Hall P: The CAGE questionnaire: Validation of a new alcoholism screening instrument. Am J Psychiatry 131:1121-1123, 1974

12. Selzer M: The Michigan Alcoholism Screening Test: The quest for a new diagnostic instrument. Am J Psychiatry 127:89-94, 1971

13. Edwards $G$ : The alcohol dependence syndrome: Usefulness of an idea, in Edwards G, Grant M (eds): Alcoholism: New Knowledge and New Responses. Baltimore, University Park Press, 1977, pp 136-156 